\title{
TRANSPORTE DO AMÔNIO EM COLUNAS COM AGREGADOS DE UM LATOSSOLO VERMELHO DISTRÓFICO
}

\author{
Fábio Cunha Coelho', Hugo Alberto Ruiz ${ }^{2}$, Paulo Afonso Ferreira ${ }^{3}$, Gonçalo Evangelista de França ${ }^{4}$, \\ Cícero Antônio de Souza Araújo ${ }^{5}$ \& Marcelo Alírio Duarte ${ }^{6}$
}

\begin{abstract}
RESUMO
Para descrever o transporte do íon amônio em diferentes classes de agregados de um Latossolo Vermelho distrófico realizou-se um experimento de laboratório cujos tratamentos corresponderam a um fatorial 3 x 5, sendo: três fontes de $\mathrm{NH}_{4}^{+}\left[\left(\mathrm{NH}_{4}\right)_{2} \mathrm{SO}_{4}, \mathrm{NH}_{4} \mathrm{Cl}\right.$ e $\left.\mathrm{NH}_{4} \mathrm{NO}_{3}\right]$ e cinco classes de agregados $(2,0-1,0 ; 1,0-0,5 ; 0,5-0,25 ; 0,25-0,105 ; \mathrm{e}<0,105 \mathrm{~mm})$. A unidade experimental foi constituída de uma coluna de vidro saturada, sob vácuo, com uma solução de $\mathrm{CaCl}_{2}, 0,005 \mathrm{~mol} \mathrm{~L}^{-1}$. Aplicou-se, a seguir, a solução saturante até percolação constante, seguida de um pulso de sete volumes de poros da respectiva fonte a $0,01 \mathrm{~mol} \mathrm{~L}^{-1} \mathrm{de} \mathrm{NH}_{4}^{+}\left(\mathrm{C}_{\mathrm{o}}\right)$ para cada classe de agregados. Foram coletadas frações correspondentes a 0,3 volume de poros do efluente (p) determinando-se as concentrações de $\mathrm{NH}_{4}^{+}(\mathrm{C})$. Calculou-se a relação $\mathrm{C} / \mathrm{C}$. para cada fração $\mathrm{p}$, traçando-se curvas experimentais de eluição. Os maiores valores dos fatores de retardamento ocorreram nos agregados de menor tamanho, indicando maior retenção do amônio; na maioria dos tratamentos ocorreu superposição das curvas obtidas por meio dos modelos teóricos, o que indicou que o transporte do amônio no solo se deu, predominantemente, por dispersão. Os ânions acompanhantes apresentaram efeitos semelhantes sobre a lixiviação do $\mathrm{NH}_{4}^{+}$, nas diferentes classes de agregados.
\end{abstract}

Palavras-chave: amônio, transporte, agregados, Latossolo Vermelho distrófico

\section{AMMONIUM TRANSPORT IN COLUMNS WITH AGGREGATES OF A TYPIC HAPLORTOX}

\begin{abstract}
To describe the transport of the ammonium ion in different classes of aggregates of a typic Haplortox, a laboratory experiment was carried out. The treatments corresponded to a $3 \times 5$ factorial, being three sources of $\mathrm{NH}_{4}^{+}\left[\left(\mathrm{NH}_{4}\right)_{2} \mathrm{SO}_{4}, \mathrm{NH}_{4} \mathrm{Cl}\right.$, and $\left.\mathrm{NH}_{4} \mathrm{NO}_{3}\right]$ and five aggregate classes $(2.0-1.0,1.0-0.5,0.5-0.25$, $0.25-0.105$, and $<0.105 \mathrm{~mm}$ ). The experimental unit was a glass column saturated, under vacuum to which was applied a $\mathrm{CaCl}_{2} 0.005$ mole $\mathrm{L}^{-1}$ solution. After saturation, the same solution was applied until constant percolation, followed by a seven pore volume pulse of solution of respective source -0.01 mole L$^{-1} \mathrm{NH}_{4}^{+}\left(\mathrm{C}_{\mathrm{o}}\right)$. Fractions of the effluent, corresponding to 0.3 pore volume (p), were collected, and the $\mathrm{NH}_{4}^{+}$concentrations were determined $(\mathrm{C})$. $\mathrm{C} / \mathrm{C}$ relationships were calculated for every $\mathrm{p}$ fraction, and the experimental elution curves were traced. The highest retardation factors were calculated for the smallest aggregates, indicating a larger retention of ammonium, and the almost superimposed theoretical curves showed the predominance of the dispersive transport for ammonium. $\mathrm{The}_{4}{ }_{4}^{+}$percolation was not affected by the studied anions in the different aggregates classes.
\end{abstract}

Key words: ammonium transport, soil aggregates, Oxysol

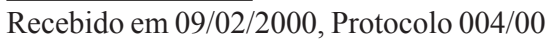

${ }^{1}$ Professor Associado - Laboratório de Fitotecnia - UENF. CEP 28015 - 620,Campos dos Goytacazes, RJ

${ }^{2}$ Professor Titular - Departamento de Solos - UFV. CEP 36571 - 000, Viçosa, MG. Bolsista do CNPq. E-mail: hruiz@mail.ufv.br

${ }^{3}$ Professor Titular - Departamento de Engenharia Agrícola - UFV. Bolsista do CNPq. Fone: (0xx31) 38992735

${ }^{4}$ Pesquisador - CNPMS/EMBRAPA. CEP 35701 - 970, Sete Lagoas, MG

${ }^{5}$ Professor - Escola Técnica Federal de Petrolina. CEP 55300 - 000, Petrolina, PE

${ }^{6}$ Bolsista de Aperfeiçoamento da FAPEMIG, Departamento de Solos - UFV
} 


\section{INTRODUÇÃO}

A baixa eficiência de fertilizantes nitrogenados em condições tropicais, tem sido atribuída ao grande potencial de perdas, sendo a lixiviação, a denitrificação e a volatilização de amônia os mecanismos mais importantes (Gamboa et al., 1971; Osiname et al., 1983).

O manejo excessivo do solo pode reduzir o tamanho dos agregados, afetando a estrutura e a dinâmica de água e de nutrientes no solo. Nos espaços interagregados, o fluxo de massa constitui o principal mecanismo de transporte de nitrogênio no solo (Santos, 1986) enquanto nos espaços intraagregados, em razão da maior resistência hidráulica, o movimento do nitrogênio se processa principalmente por difusão. Cornforth (1968) observou maior absorção de nitrogênio por gramíneas em agregados do solo com diâmetros médios de 1 a $10 \mathrm{~mm}$ e, segundo ele, a menor absorção de nitrogênio nos agregados maiores que $10 \mathrm{~mm}$ poderia ser atribuída à restrição na absorção de água pelas raízes das plantas no interior dos agregados, enquanto nos agregados menores que $1 \mathrm{~mm}$ a menor absorção do nitrogênio poderia ser atribuída a problemas de aeração do meio; assim, no interior dos agregados maiores que $10 \mathrm{~mm}$, em razão da resistência hidráulica, e nos agregados menores que $1 \mathrm{~mm}$, em razão dos problemas de aeração, poderia ter havido perdas de nitrogênio por denitrificação que, segundo Stanford \& Epstein (1974) podem ocorrer quando o potencial da água no solo é superior a $-10 \mathrm{kPa}$ ou quando mais de $90 \%$ do espaço poroso do solo se encontra ocupado por água.

Em condições de maior permanência da água da chuva ou da irrigação, o nitrogênio adicionado ao solo pode difundir-se para o interior dos agregados maiores, circunstâncias em que, apesar das restrições na absorção do nutriente pela planta e do aumento do processo de desnitrificação, a lixiviação do nitrogênio na forma de nitrato pode ser bastante reduzida (Wiersum, 1962; Addiscott et al., 1983).

Diversos modelos matemáticos, baseados em curvas de efluente, têm sido propostos para descrever o transporte de solutos no solo, dentre os quais podem ser citados os propostos por Kirkham \& Powers (1972). O formato e a posição das curvas de efluente dependem, para um mesmo tipo de solo, do tamanho dos agregados e da magnitude da velocidade convectiva. Nielsen \& Biggar (1961) e Biggar \& Nielsen (1967) trabalhando com classes de agregados de solo de 0,25 a 0,$5 ; 0,5$ a 1,0 ; e de 1,0 a 2,0 mm constataram, após o deslocamento de uma solução de $\mathrm{CaCl}_{2}$ com uma de $\mathrm{CaSO}_{4}$, que as curvas de efluente se tornavam mais horizontais com o aumento do tamanho dos agregados, ou seja, os valores da relação entre as concentrações da solução após a eluição e as concentrações da solução antes da eluição $\left(\mathrm{C} / \mathrm{C}_{\mathrm{o}}\right)$ eram menores quando a percolação se processava nos agregados maiores; também as curvas de efluente se deslocavam para a direita, a medida em que a velocidade convectiva do fluido aumentava, com aumento mais tardio da concentração do efluente.

O objetivo deste trabalho foi descrever o transporte do amônio em colunas com diferentes classes de agregados de um Latossolo Vermelho distrófico, quando aplicado na forma de sulfato, nitrato ou cloreto, por comparação dos coeficientes de retardamento do amônio e pelo ajuste de dois modelos teóricos que consideram o transporte por difusão e por dispersão.

\section{MATERIAL E MÉTODOS}

A amostra de solo foi retirada de um Latossolo Vermelho distrófico (LVd), A moderado, textura argilosa, fase relevo suave ondulado, cerrado, sob área limpa, previamente cultivada com milho durante vários anos, localizada no Centro Nacional de Pesquisa do Milho e Sorgo/EMBRAPA, Sete Lagoas, MG. Foi composta de quatro amostras simples retiradas em locais diferentes da área, na profundidade de $0-10 \mathrm{~cm}$. Realizou-se um experimento de laboratório cujos tratamentos corresponderam a um fatorial $3 \times 5$, sendo: três fontes de $\mathrm{NH}_{4}^{+}\left(\left(\mathrm{NH}_{4}\right)_{2} \mathrm{SO}_{4}\right.$, $\mathrm{NH}_{4} \mathrm{Cl}$ e $\mathrm{NH}_{4} \mathrm{NO}_{3}$ ) e cinco classes de agregados (2,0 a 1,0; 1,0 a $0,5 ; 0,5 \mathrm{a} 0,25 ; 0,25 \mathrm{a} 0,105 ; \mathrm{e}<0,105 \mathrm{~mm})$. A unidade experimental foi constituída de uma coluna de vidro de $2 \mathrm{~cm}$ de diâmetro interno e $25 \mathrm{~cm}$ de comprimento, com a extremidade inferior afunilada e conectada a uma mangueira de látex, com pinça de Hoffmann para regular a vazão. Foi utilizada lã de vidro sobreposta a uma tela plástica na parte inferior para se evitar qualquer perda de solo, enquanto as colunas foram preenchidas com agregados até os $5 \mathrm{~cm}$ da borda superior sendo cobertos com lã de vidro para evitar o contato direto com o fluido deslocador. O delineamento experimental foi em blocos casualizados, com três repetições.

As cinco classes de agregados foram separadas por peneiramento a seco e caracterizadas, determinando-se o $\mathrm{pH}$ em água (EMBRAPA, 1979), a capacidade de troca catiônica efetiva e total (EMBRAPA, 1979), o carbono orgânico pelo método de Walkley \& Black (Jackson, 1958), a composição granulométrica pelo método da pipeta (EMBRAPA, 1979), a densidade dos agregados secos e empacotados conforme método da proveta para densidade do solo (EMBRAPA, 1979) e a densidade das partículas pelo método do balão volumétrico (EMBRAPA, 1979).

Tabela 1. Caracterização química e física dos agregados do Latossolo Vermelho-Escuro

\begin{tabular}{lccccc}
\hline \multirow{2}{*}{ Característica } & \multicolumn{5}{c}{ Classe de Agregados } \\
\cline { 2 - 6 } & $\mathrm{A}$ & $\mathrm{B}$ & $\mathrm{C}$ & $\mathrm{D}$ & $\mathrm{E}$ \\
\hline $\mathrm{pH}$ em água $(1: 2,5)$ & 5,2 & 5,1 & 5,1 & 5,0 & 5,0 \\
CTC efetiva $\left(\mathrm{mmol}_{\mathrm{c}} \mathrm{dm}^{-3}\right)$ & 23 & 26 & 28 & 29 & 28 \\
CTC total $\left(\mathrm{mmol}_{\mathrm{c}} \mathrm{dm}^{-3}\right)$ & 93 & 99 & 111 & 114 & 117 \\
Carbono orgânico $\left(\mathrm{g} \mathrm{kg}^{-1}\right)$ & 25 & 28 & 32 & 33 & 40 \\
Granulometria $\left(\mathrm{kg} \mathrm{kg}^{-1}\right)$ & & & & & \\
$\quad$ Areia & 0,31 & 0,25 & 0,17 & 0,14 & 0,04 \\
$\quad$ Silte & 0,16 & 0,20 & 0,27 & 0,24 & 0,27 \\
$\quad$ Argila & 0,53 & 0,55 & 0,56 & 0,62 & 0,69 \\
Densidade $\left(\mathrm{kg} \mathrm{dm}^{-3}\right)$ & & & & & \\
$\quad$ Aparente & 0,92 & 0,94 & 0,93 & 0,91 & 0,90 \\
$\quad$ Partículas & 2,50 & 2,56 & 2,52 & 2,47 & 2,36 \\
\hline $\begin{array}{l}\text { As classes A,B,C,D e E referem agregados de tamanho entre 2,0-1,0;1,0-0,5; } 0,5-0,25 ; 25-0,105 \mathrm{e} \\
<0,105 \text { mm }\end{array}$
\end{tabular}

As colunas contendo os agregados foram fixadas verticalmente numa estrutura apropriada, submetidas a duas horas de vácuo e, a seguir, lentamente, de baixo para cima, saturadas com uma solução de $\mathrm{CaCl}_{2} 0,005 \mathrm{~mol} \mathrm{~L}^{-1}$, usando-se um conjunto de alimentação automático (frasco de Mariotte); terminada a saturação, o sistema de alimentação foi acondicionado na extremidade superior da coluna, deixandose percolar a solução de $\mathrm{CaCl}_{2}$ até obtenção de velocidade constante na eluição, uniformizada e próximo daquela das colunas com agregados de menor tamanho; posteriormente, 
aplicou-se um pulso de sete volumes de poros de $\left(\mathrm{NH}_{4}\right)_{2} \mathrm{SO}_{4}$ ou $\mathrm{NH}_{4} \mathrm{Cl}$ ou $\mathrm{NH}_{4} \mathrm{NO}_{3}$ a $0,01 \mathrm{~mol} \mathrm{~L}^{-1}{\mathrm{de} \mathrm{NH}_{4}}^{+}\left(\mathrm{C}_{\mathrm{o}}\right)$ para cada classe de agregados, quando o nível da solução de $\mathrm{CaCl}_{2}$ desceu até a extremidade da coluna de agregados. Foram coletadas frações correspondentes a 0,3 volume de poros do efluente (p). Determinaram-se, nas soluções efluentes, as concentrações de $\mathrm{NH}_{4}^{+}$(C) (Alves et al., 1993) e, com o valor de C, calculou-se a relação $\mathrm{C} / \mathrm{C}_{\mathrm{o}}$ para cada fração $\mathrm{p}$, traçando-se curvas experimentais de eluição.

$\mathrm{O}$ volume de poros de cada coluna $\left(\mathrm{V}_{\mathrm{p}}\right)$ foi determinado pela relação:

$$
\mathrm{V}_{\mathrm{p}}=\pi \mathrm{r}^{2} \mathrm{~h}\left(1-\frac{\mathrm{D}_{\mathrm{s}}}{\mathrm{D}_{\mathrm{p}}}\right)
$$

em que:

$$
\begin{array}{ll}
\mathrm{r} & \text { - raio da coluna }(1 \mathrm{~cm}) \\
\mathrm{h} & \text { - comprimento da coluna }(20 \mathrm{~cm}) \\
\mathrm{D}_{\mathrm{s}} & \text { - densidade aparente }\left(\mathrm{kg} \mathrm{dm}^{-3}\right) \\
\mathrm{D}_{\mathrm{p}} & \text { - densidade das partículas }\left(\mathrm{kg} \mathrm{dm}^{-3}\right)
\end{array}
$$

Para se caracterizar o transporte de amônio nos agregados, utilizou-se um modelo que considera apenas a dispersão e um outro, que leva em conta a transferência de soluto por difusão e dispersão (Kirkham \& Powers, 1972) descritos a seguir.

Modelo que considera apenas a dispersão (Modelo 1):

$$
\frac{\mathrm{C}}{\mathrm{C}_{\mathrm{o}}}=\frac{1}{2}\left\{\operatorname{erfc}\left[\frac{\mathrm{R}-\mathrm{p}}{2(\mathrm{ERp} / \mathrm{vL})^{1 / 2}}\right]\right\}
$$

Modelo que considera a transferência de soluto por difusão e por fluxo de massa (Modelo 2):

$$
\frac{\mathrm{C}}{\mathrm{C}_{\mathrm{o}}}=\frac{1}{2}\left\{\operatorname{erfc}\left[\frac{\mathrm{R}-\mathrm{p}}{2(\mathrm{DRp} / \mathrm{vL})^{1 / 2}}\right]+\mathrm{e}^{\mathrm{vL} / \mathrm{D}} \operatorname{erfc}\left[\frac{\mathrm{R}+\mathrm{p}}{2(\mathrm{DRp} / \mathrm{vL})^{1 / 2}}\right]\right\}
$$

em que:

C - concentração do soluto $\left(\mathrm{kg} \mathrm{dm}^{-3}\right)$

$\mathrm{C}_{\mathrm{o}}$ - concentração do soluto no fluido deslocador $\left(\mathrm{kg} \mathrm{dm}^{-3}\right)$

erfc - função erro complementar $=1$ - erf

erf - função erro

$\mathrm{p}$ - número de volume de poros (adimensional)

E - coeficiente de dispersão $\left(\mathrm{cm}^{2} \mathrm{~h}^{-1}\right)$ calculado pela fórmula: $\mathrm{E}=\mathrm{vL} / 4 \mathrm{pS}^{2}$

$\mathrm{S}$ - declividade da curva experimental a um volume de poros correspondente a $\mathrm{C} / \mathrm{C}_{\mathrm{o}}=0,5$ (adimensional)

$\mathrm{V}$ - velocidade de avanço $\left(\mathrm{cm} \mathrm{h}^{-1}\right)$

L - comprimento da coluna de solo $(\mathrm{cm})$

D - coeficiente de difusão $\left(\mathrm{cm}^{2} \mathrm{~h}^{-1}\right)$ obtido pela expressão: $\mathrm{D}=\mathrm{vL} /\left(4 \mathrm{pS}^{2}\right)$.

Como o material de solo utilizado adsorve amônio, incluiu-se o fator de retardamento $(\mathrm{R})$ nos modelos teóricos, o qual foi estimado ajustando-se equações de regressão aos dados experimentais e se verificando o valor $\mathrm{p}=\mathrm{R}$ a $\mathrm{C} / \mathrm{C}_{\mathrm{o}}=0,5$ (van Genuchten \& Wierenga, 1986). Obtiveram-se, a seguir, as curvas teóricas de eluição e, com os valores dos fatores de retardamento, realizou-se a análise de variância seguida de ajustes de equações de regressão em função do diâmetro médio das classes de agregados e de teste de contrastes entre as fontes de $\mathrm{NH}_{4}^{+}$.

\section{RESULTADOS E DISCUSSÃO}

Foram calculadas a velocidade de avanço das soluções eluídas nas colunas, as declividades das curvas de eluição experimentais para $\mathrm{C} / \mathrm{C}_{\mathrm{o}}=0,5$, o fator de retardamento e o coeficiente dispersivo-difusivo (Tabela 2).

Tabela 2. Velocidade de avanço (v), declividade da curva de eluição experimental a $\mathrm{C} / \mathrm{C}_{\mathrm{o}}=0,5(\mathrm{~S})$, fator de retardamento (R) e coeficiente dispersivo-difusivo (D) para diferentes sais de amônio, considerando-se a classe de agregados

\begin{tabular}{ccccc}
\hline $\begin{array}{c}\text { Agregados } \\
(\mathrm{mm})\end{array}$ & $\begin{array}{c}\mathrm{V} \\
\left(\mathrm{cm} \mathrm{h}^{-1}\right)\end{array}$ & $\mathrm{S}$ & $\mathrm{R}$ & $\begin{array}{c}\mathrm{D} \\
\left(\mathrm{cm}^{2} \mathrm{~h}^{-1}\right)\end{array}$ \\
\hline \multicolumn{5}{c}{ Sulfato de Amônio } \\
$2,0-1,0$ & 3,4 & 0,4 & 2,5 & 42,3 \\
$1,0-0,5$ & 3,5 & 0,4 & 2,9 & 45,5 \\
$0,5-0,25$ & 3,5 & 0,3 & 3,4 & 58,3 \\
$0,25-0,105$ & 3,5 & 0,3 & 3,2 & 50,9 \\
$<0,105$ & 3,3 & 0,3 & 2,9 & 58,6 \\
\hline \multicolumn{5}{c}{ Cloreto de Amônio } \\
$2,0-1,0$ & 3,7 & 0,3 & 2,4 & 51,2 \\
$1,0-0,5$ & 3,4 & 0,5 & 2,6 & 24,2 \\
$0,5-0,25$ & 3,5 & 0,4 & 2,8 & 31,4 \\
$0,25-0,105$ & 3,8 & 0,3 & 3,1 & 58,9 \\
$<0,105$ & 2,9 & 0,3 & 2,9 & 57,7 \\
\hline \multicolumn{5}{c}{ Nitrato de Amônio } \\
$2,0-1,0$ & 3,5 & 0,4 & 2,6 & 45,8 \\
$1,0-0,5$ & 4,4 & 0,4 & 2,3 & 43,9 \\
$0,5-0,25$ & 3,7 & 0,4 & 3,0 \\
$0,25-0,105$ & 3,8 & 0,4 & 2,8 & 40,9 \\
$<0,105$ & 3,0 & 0,3 & 3,0 \\
\hline \multicolumn{5}{c}{}
\end{tabular}

Pela análise de variância seguida de testes de contraste, não houve diferença significativa para os valores dos coeficientes de retardamento em relação ao ânion acompanhante das fontes de $\mathrm{NH}_{4}^{+}$utilizadas; portanto, os ânions apresentaram efeito semelhante nos processos de adsorção e lixiviação do $\mathrm{NH}_{4}^{+}$, nas diferentes classes de agregados (Tabela 2).

Os maiores valores dos fatores de retardamento ocorreram nos agregados de menor tamanho (Figura 1) indicando que, nesses, houve maior retenção do amônio, cuja característica estaria relacionada à maior área de exposição de sítios de adsorção devido à maior área específica, a medida em que a partícula é menor, e ao maior teor de matéria orgânica e argila nesses agregados (Tabela 1) o que levaria a uma capacidade maior de sorção catiônica. Resultado semelhante foi observado por Araújo (1997) para os íons $\mathrm{K}^{+}$e $\mathrm{H}_{2} \mathrm{PO}_{4}^{-}$, em experimento com colunas preenchidas com agregados do mesmo $\mathrm{LVd}$ utilizado neste ensaio. Os resultados obtidos para o amônio, no ensaio, indicam que no caso de se utilizar a fertirrigação como meio para fornecer o nitrogênio às plantas em um programa de adubação, em LVd com maior proporção de agregados de menor tamanho, haveria necessidade de se 


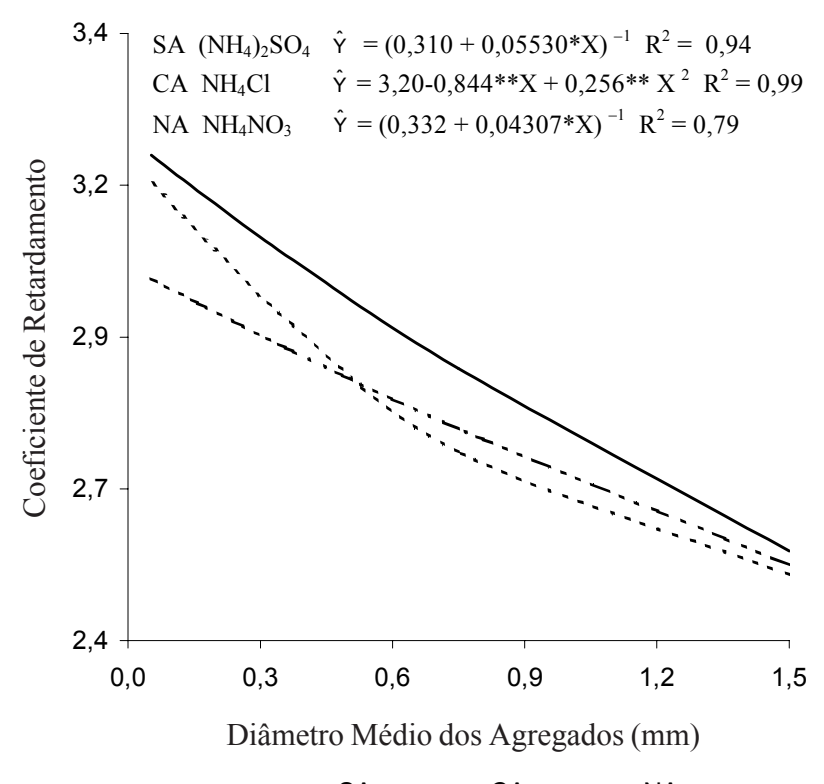

Figura 1. Coeficientes de retardamento em relação ao diâmetro médio dos agregados, considerando-se a solução de eluição

determinar o momento de aplicar a lâmina de água contendo o $\mathrm{NH}_{4}^{+}$de modo que, após a sua aplicação, a irrigação continuaria objetivando transportar o nutriente até a profundidade onde se encontra o maior volume de raízes ativas (Coelho, 1994). Vale ressaltar, ainda, que independentemente do tamanho dos agregados, o sulfato de amônio foi o sal que apresentou os maiores valores do fator de retardamento indicando, assim, menor mobilidade no solo comparativamente ao nitrato e ao cloreto de amônio. Relativamente ao cloreto de amônio, os valores do fator de retardamento mostraram-se intermediários para agregados $<0,6 \mathrm{~mm}$ e mínimos quando os agregados eram $>0,6 \mathrm{~mm}$. Por outro lado, o nitrato de amônio apresentou valores mínimos do fator de retardamento para diâmetros de agregados $<0,6 \mathrm{~mm}$ e valores intermediários para diâmetros $>0,6 \mathrm{~mm}$. A Figura 1 apresenta, também, a tendência das curvas de regressão a convergirem-se à medida que o tamanho dos agregados tende para $1,5 \mathrm{~mm}$, o que caracteriza comportamento semelhante dos três sais de amônio, quanto à mobilidade no solo, nos maiores agregados.

Na maioria dos tratamentos houve superposição das curvas de eluição obtidas por meio dos modelos teóricos, o que indicou que o transporte do amônio no solo foi predominantemente por dispersão (Figuras 2, 3 e 4). A diferença entre os modelos 1 e 2 está relacionada ao efeito da difusão; conseqüentemente, como houve justaposição das curvas, este fenômeno foi insignificante.

A predominância da dispersão no transporte do amônio era esperada; no entanto, esperava-se também alguma contribuição da difusão nos agregados maiores, de forma a explicar o movimento do cátion da superfície dos agregados para o interior, assim como já foi observado para o nitrato, por van Genuchten \& Wierenga (1986). Os resultados obtidos confirmam o trabalho de Antonopoulos (1993) que testou um modelo baseado no método de elementos finitos, no qual considerou o transporte de amônio por dispersão, a nitrificação e a desnitrificação, concluindo que o transporte do amônio no solo foi predominantemente por dispersão. Seu modelo foi validado
A. Agregados entre 2,0 e 1,0 mm

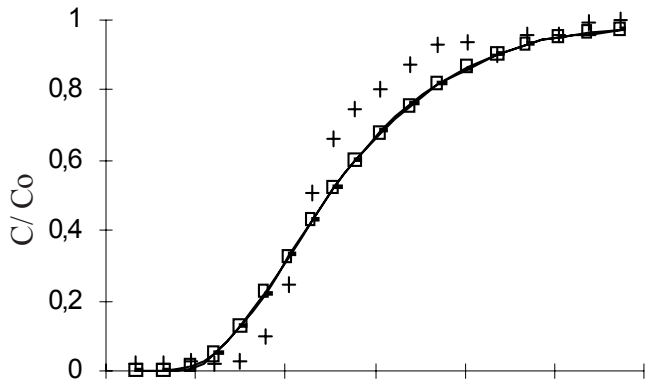

B. Agregados entre 1,0 e $0,5 \mathrm{~mm}$

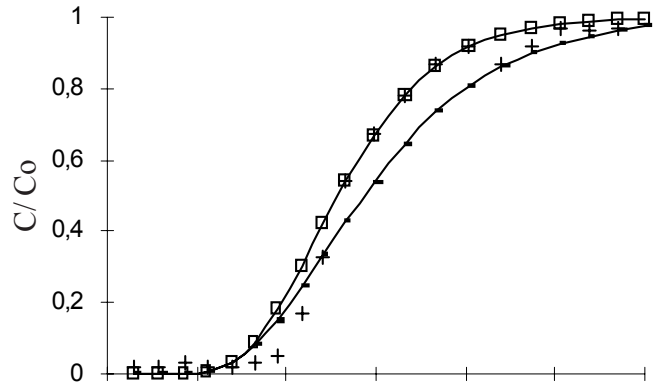

C. Agregados entre 0,5 e 0,25 mm

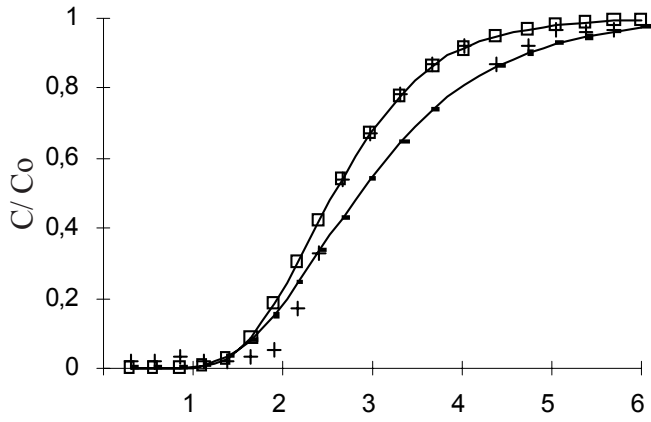

D. Agregados entre 0,25 e 0,105 mm

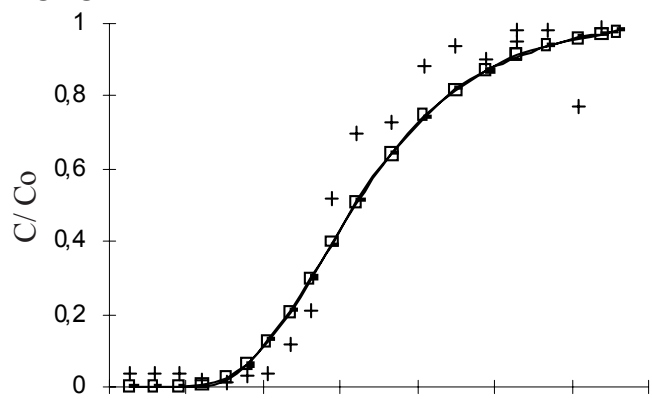

E. Agregados $<0,105 \mathrm{~mm}$

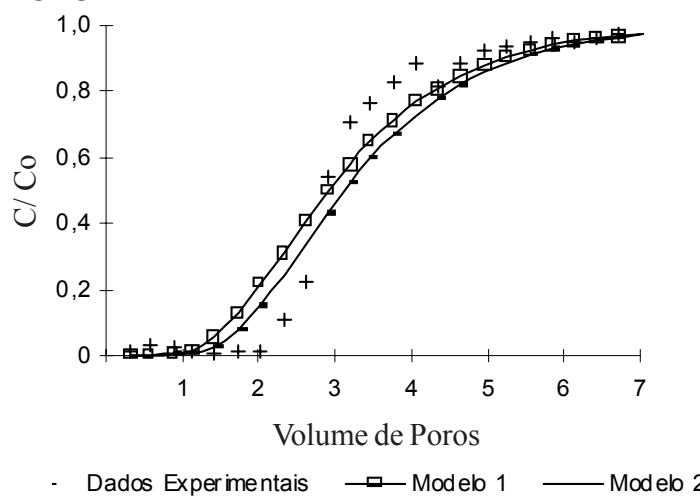

Figura 2. Curvas de eluição para amônio, para os dados experimentais e calculados pelos modelos 1 e 2, para cada classe de agregados, cuja fonte foi o sulfato de amônio 
A. Agregados entre 2,0 e 1,0 mm

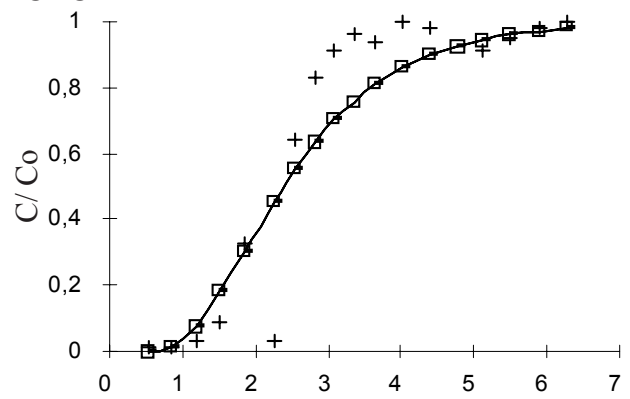

B. Agregados entre 1,0 e $0,5 \mathrm{~mm}$

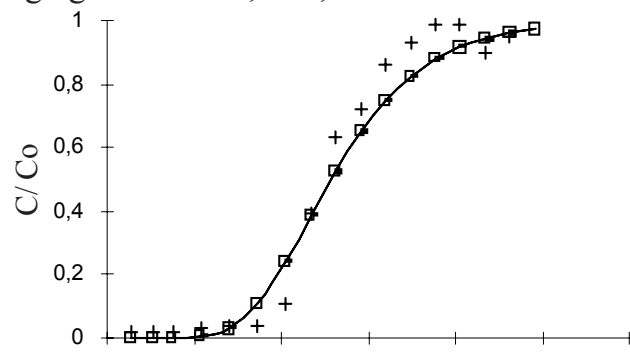

C. Agregados entre 0,5 e $0,25 \mathrm{~mm}$

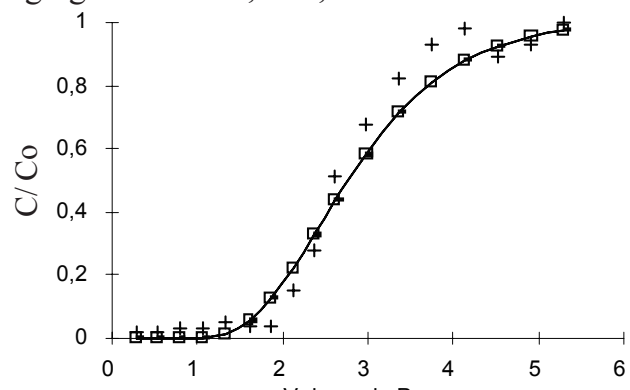

D. Agregados entre 0,25 e $0,105 \mathrm{~mm}$

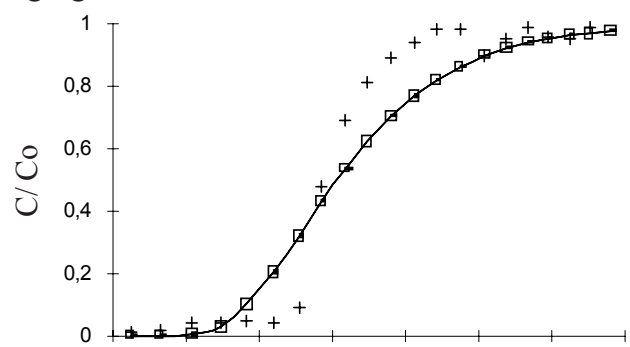

E. Agregados $<0,105 \mathrm{~mm}$

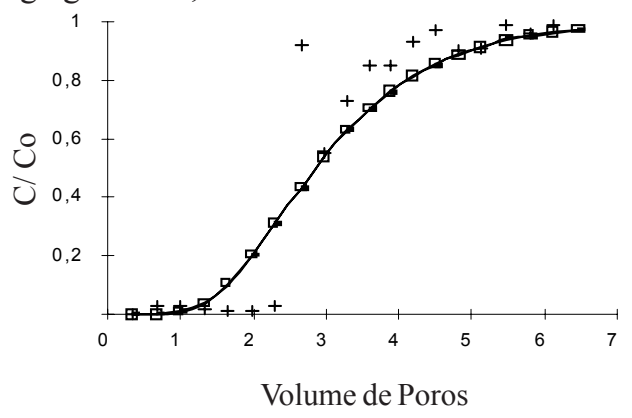

+ Dados Experimentais - Modelo $1-$ Modelo 2

Figura 3. Curvas de eluição para amônio, para os dados experimentais e calculados pelos modelos 1 e 2, para cada classe de agregados, cuja fonte foi o cloreto de amônio

para diferentes solos sob regime de escoamento em meio saturado, porém resultados contrastantes são encontrados na literatura quanto à predominância do fluxo de massa no transporte de amônio. Teo et al. (1994) em experimento de campo
A. Agregados entre 2,0 e 1,0 mm

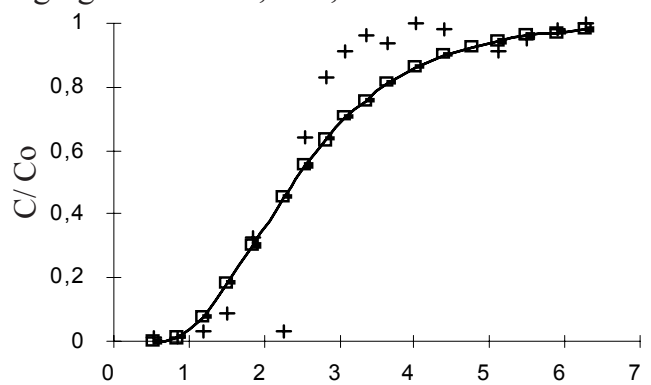

B. Agregados entre 1,0 e $0,5 \mathrm{~mm}$

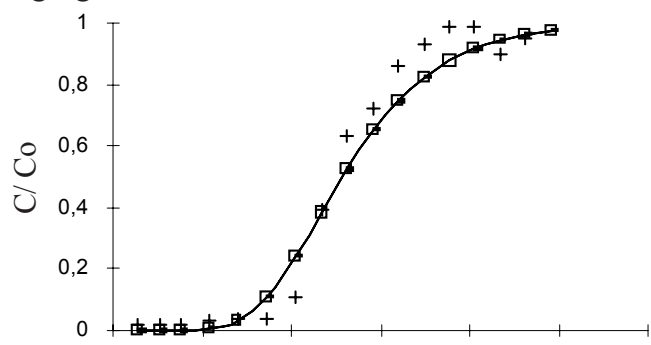

C. Agregados entre 0,5 e $0,25 \mathrm{~mm}$

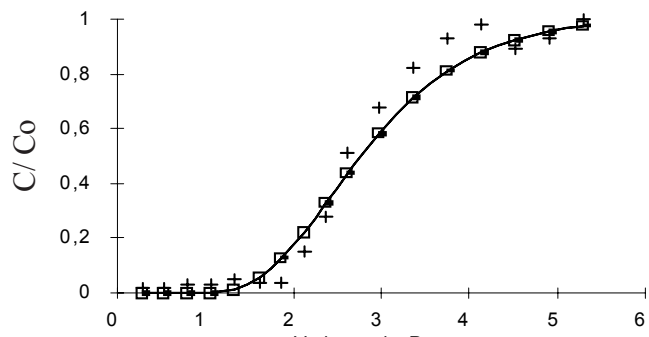

D. Agregados entre $0,25 \mathrm{e} 0,105 \mathrm{~mm}$
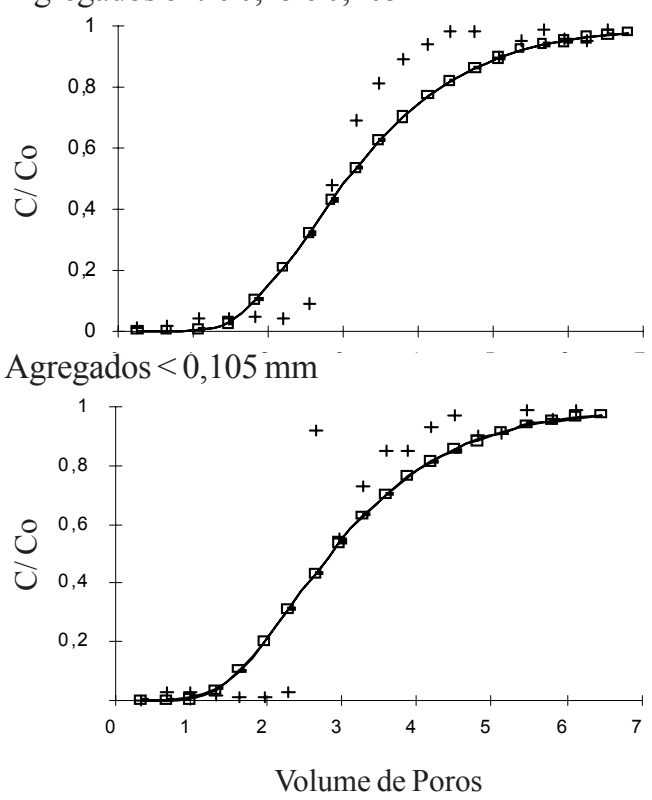

+ Dados Experimentais $\square$ Modelo 1 Modelo 2

Figura 4. Curvas de eluição para amônio, para os dados experimentais e calculadas pelos modelos 1 e 2 , para cada classe de agregados, cuja fonte foi o nitrato de amônio

realizado em Stuttgart, Arkansas (EUA) em condições de cultivo de arroz irrigado por inundação, determinaram a importância relativa do fluxo de massa e da difusão no movimento de macronutrientes até as raízes do arroz, e concluíram, ainda, que a difusão foi o fator dominante que controlou a disponibilidade de $\mathrm{NH}_{4}^{+}$, P, e K nos solos estudados, sob regime de inundação. 
Observando-se as curvas de eluição (Figuras 2, 3 e 4) nota-se que, na fase inicial de eluição (até aproximadamente 2,5 volumes de poros) ocorreu maior retardamento do $\mathrm{NH}_{4}^{+}$, certamente pela maior taxa de adsorção, no momento em que os sítios de adsorção ainda não estavam saturados por este cátion, condições em que, praticamente em todas as classes de agregados e soluções de eluição avaliadas, os modelos teóricos tenderam a superestimar os valores de $\mathrm{C} / \mathrm{C}_{\mathrm{o}}$, enquanto, a partir deste valor, houve uma subestimação da relação $\mathrm{C} / \mathrm{C}_{\mathrm{o}}$. Araújo (1997) também observou que esses mesmos modelos teóricos superestimaram os valores de $\mathrm{C} / \mathrm{C}_{\mathrm{o}}$ para agregados do mesmo LVd eluído com solução de $\mathrm{KH}_{2} \mathrm{PO}_{4}$, não sendo verificada, entretanto, nenhuma região em que os modelos teóricos subestimaram os valores de $\mathrm{C} / \mathrm{C}_{\mathrm{o}}$.

\section{CONCLUSÕES}

1. Os maiores valores dos fatores de retardamento ocorreram nos agregados de menor tamanho, indicando que, nesses agregados, há maior retenção do amônio.

2. O transporte do amônio ocorreu predominantemente por dispersão.

3. Os ânions acompanhantes apresentaram efeitos semelhantes sobre os processos de adsorção e lixiviação do $\mathrm{NH}_{4}^{+}$, nas diferentes classes de agregados.

\section{REFERÊNCIAS BIBLIOGRÁFICAS}

ADDISCOTT, T.M.; THOMAS, V.H.; JANJUA, M.A. Measurement and simulation of anion diffusion in natural soil aggregates and clods. Journal of Soil Science, Reading, v.34, p.709-721, 1983.

ALVES, B.J.R.; BODDEY, R.M.; URQUIAGA, S.S. A rapid and sensitive flow injection technique for the analysis of ammonium in soil extracts. Communications in Soil Science and Plant Analysis, New York, v.24, p.277-284, 1993.

ANTONOPOULOS, V.Z. Simulation of water and nitrogen dynamics in soils during waste water applications by using a finite-element model. Water Resources Management, Dordrecht, v.7, p.237-251, 1993.

ARAUJO, C.A.S. Movimento de fósforo e de macronutrientes catiônicos em agregados de um Latossolo Vermelho-Escuro. Viçosa, UFV, 1997. 124p. Tese Doutorado
BIGGAR, J.W.; NIELSEN, D.R. Miscible displacement and leaching phenomenon. In: HAGAN, R.M.; HAISE, H.R. e EDMINSTER, T.W. (ed.). Irrigation of agricultural lands. Madison: American Society of Agronomy, 1967. p.254-274.

COELHO, A.M. Fertirrigação. In: COSTA, E.F.; VIEIRA, R.F. e VIANA, P.A. (ed.). Quimigação - Aplicação de produtos químicos e biológicos via irrigação. Brasília: EMBRAPA SPI, 1994. 315 p.

CORNFORTH, I.S. The effect of the size of soil aggregates on nutrient supply. Journal of Agricultural Science, London, v.70, p.83-85, 1968.

EMBRAPA. Manual de métodos de análise de solo. Rio de Janeiro: Serviço Nacional de Levantamento e Conservação de Solos, 1979. s.n.p.

GAMBOA, J.; PEREZ, G.; BLASCO, M. Un modelo para describir los procesos de retención y lixiviación en los suelos. Turrialba, Costa Rica, 21:312-316, 1971.

JACKSON, M.L. Soil chemical analysis. New Jersey: PrenticeHall, 1958. p. 205-226.

KIRKHAM, D.; POWERS, W. L. Advanced soil physics. New York: John Wiley-Interscience, 1972. 534p.

NIELSEN, D.R.; BIGGAR, J.W. Miscible displacement in soils. I. experimental information. Soil Science Society of America Proceeding, Madison, v.25, p.1-5, 1961.

OSINAME, O.; GIJN VAN, H.; VLEK, P.L.G. Effect of nitrification inhibitors on the fate and efficiency of nitrogenous fertilizers under simulated humid tropical conditions. Tropical Agriculture, Trinidad \& Tobago, v.60, p.211-217, 1983.

SANTOS, C.R. Movimento vertical de nitrato, amônio, cloreto e potássio em colunas de solo irrigado. Viçosa, UFV, 1986. 66p. Dissertação Mestrado.

STANFORD, G.; EPSTEIN, E. Nitrogen mineralization-water relations in soils. Soil Science Society of America Proceeding, Madison, v.38, p.103-107, 1974.

TEO, Y.H.; BEYROUTY, C.A.; NORMAN, R.J.; GBUR, E.E. Nutrient supplying capacity of a paddy rice soil. Journal of Plant Nutrition, New York, v.17, p.1983-2000, 1994.

van GENUCHTEN, M.Th.; WIERENGA, P.J. Solute dispersion: coefficients and retardation factors. In: KLUTE, A, (ed.). Methods of soil analysis. Part 1. Physical and mineralogical properties, including statistics of measurements and sampling. $2^{\text {nd }}$ ed. Madison: American Society of Agronomy, Soil Science Society of America, 1986. p. 1025-1031.

WIERSUM, L.K. Uptake of nitrogen and phosphorus in relation to soil structure and nutrient mobility. Plant and Soil, The Hague, v.16, p.62-69, 1962. 